\title{
Safety and efficacy of neoadjuvant chemotherapy for cervix adenocarcinoma during pregnancy: case report
}

\author{
Resende, LSA $^{1}$ and Teixeira, $\mathrm{M}^{2}$ \\ ${ }^{1}$ Hospital de Base, Brasília - DF, Brazil ${ }^{2}$ Hospital Sírio Libanês, Brasília - DF, Brazil \\ leandroresende@yahoo.com.br
}

Intoduction: Cervical cancer (CC) is the $3^{\text {rd }}$ most prevalent cancer worldwide and $2^{\text {nd }}$ most prevalent in women 44 years or younger ${ }^{1}$. Each year 530.000 new cases of cervical cancer have been reported worldwide ${ }^{2}$. Cervical adenocarcinoma represents $10-20 \%$ of cases $^{3}$. The treatment of CC during pregnancy is complex and not clear. The recommended neoadjuvant therapy (NACT) regimen is platinumbased drug for the treatment of this type of cancer during pregnancy, even with possibility of intrauterine growth restriction to the fetus, besides toxicity ${ }^{7}$. It is necessary to share information and responsibility with the patient and family, in addition to the multidisciplinary team, including the obstetrician and take into consideration the optimal oncologic therapy as well as the preservation of the health of the fetus ${ }^{6}$. Treatment options include conservative and surgical approaches based on tumor size, lymph node involvement, gestational age, histological type and the patient's wish to continue the pregnancy. We present an 18-week pregnancy cervical adenocarcinoma case with favorable outcomes after neoadjuvant chemotherapy. Case presentation: A 40-year-old woman, G2P1, 18 weeks, BMI $31 \mathrm{~kg} / \mathrm{m}^{2}$; referred due recurrent vaginal bleeding. A polypoid lesion in the cervix was observed, measuring $30 \mathrm{~mm}$ which biopsy evidenced focal, well differentiated invasive endocervical adenocarcinoma. Physical and image examination using Magnetic Resonance imaging (MRI) confirmed lesion restricted to the cervix, with no parametrial commitment (Fig.1) and no distant metastasis was detected by CT scan; staged IB1 (FIGO). After oncologist evaluation was decided to maintain the pregnancy and start chemotherapy after signing a free and informed consent form. The patient received neoadjuvant chemotherapy with 4 cycles of cisplatin (CDDP; $50 \mathrm{mg} / \mathrm{m} 2$ ) every 3 weeks. Any obstetric complications during pregnancy; performed a new MRI at 32 weeks, with no changes in tumor (Fig.2). Fetal growth assessment was evaluated every 2 weeks; cesarean section at 34 weeks. A female newborn, weighed $2.400 \mathrm{~g}$, Apgar scores 7/8; admitted at the Neonatal Intensive Care Unit for respiratory distress syndrome for 3 days, went home on $5^{\text {th }}$ day. After the cesarean section, patient underwent radical hysterectomy with pelvic lymphadenectomy. We need to perform bilateral clamping of the uterine arteries prior to pelvic lymphadenectomy due to excessive bleeding. Final histological result confirmed endocervical adenocarcinoma (G1). Stromal invasion: depth $10 \mathrm{~mm}$ and extension $15 \mathrm{~mm}$. The tumor had free surgical margins, no lymph vascular space invasion and negative pelvic lymph nodes. Follow up with no recurrence for 2 years.

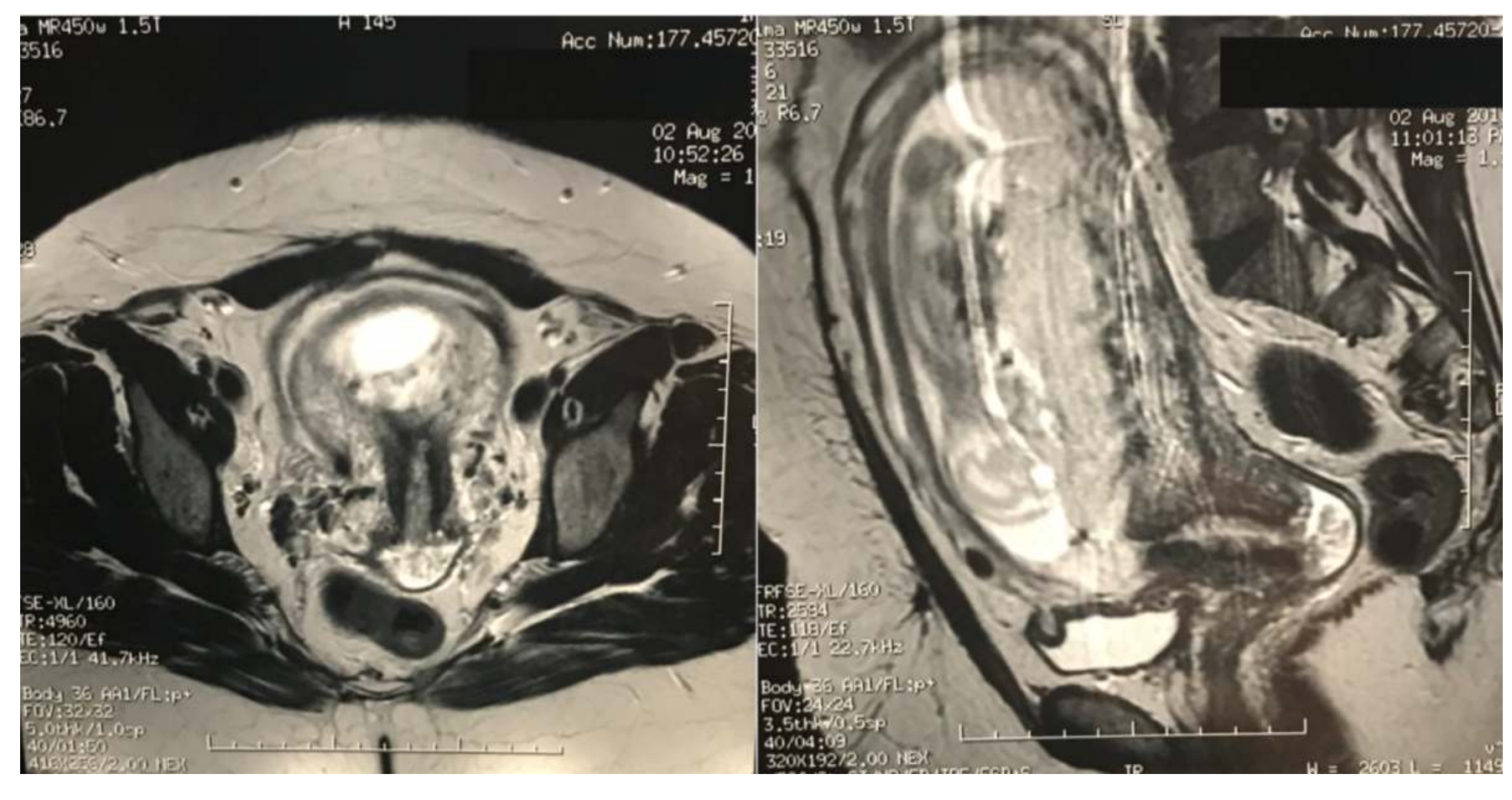

Fig.1. MRI pre neoadjuvant chemotherapy: with utero gravidarum, single fetus. Exophytic lesion involving the anterior lip of the uterine cervix, measuring $23 \times 23 \times 11 \mathrm{~mm}$, extending to the right posterolateral right wall of the vagina in the upper third, but without signs of infiltration.

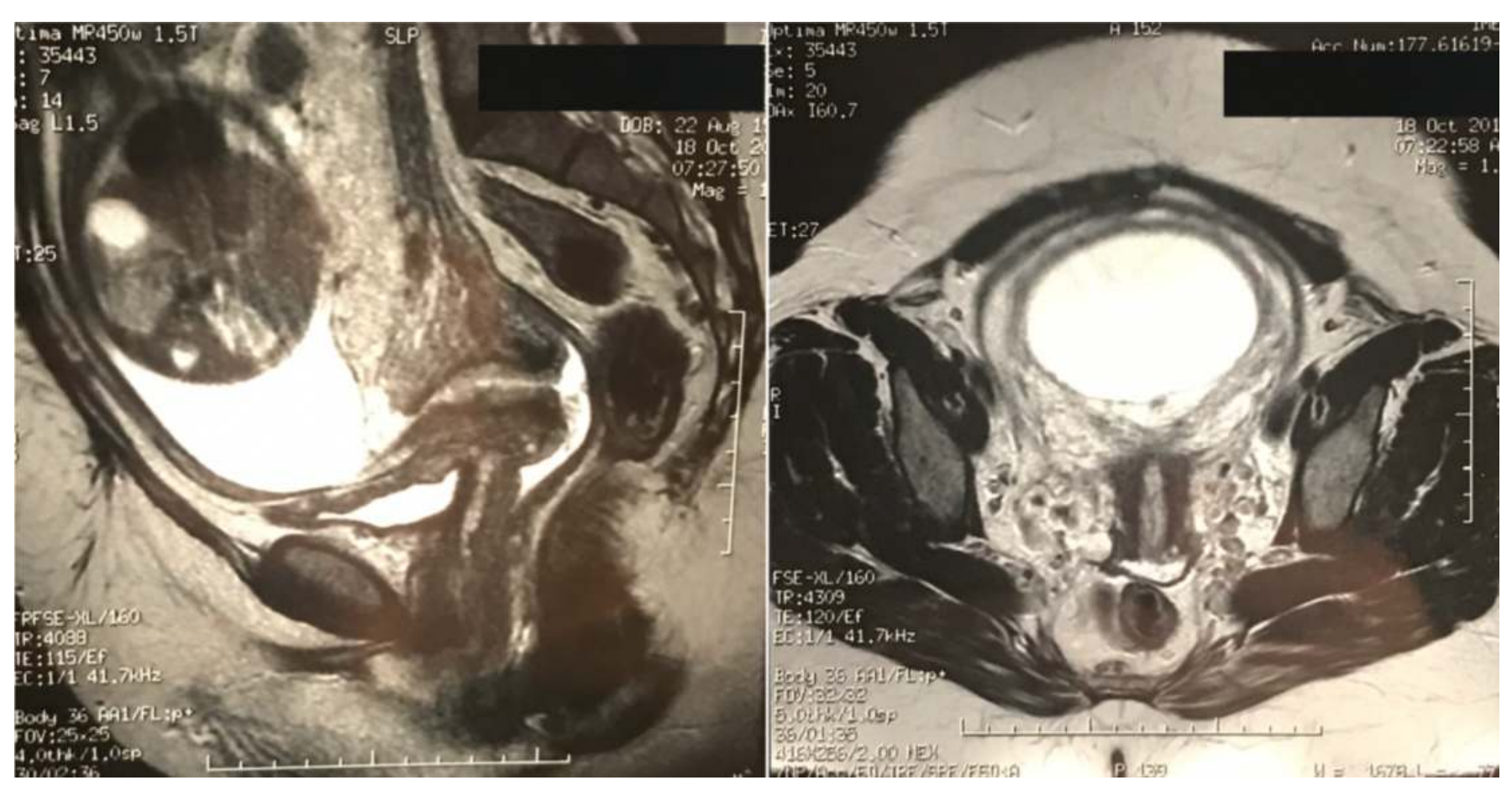

Fig. 2. MRI after neoadjuvant chemotherapy: with pregnant utero, single fetus. Exophytic lesion, compromising anterior cervical lip, compatible with primary neoplastic involvement, with minimal reduction of dimensions compared to previous examination.

Discussion: The treatment of CC during pregnancy still has limitations in order to reduce fetal risk. Cisplatin as NACT for IB1 CC was safe for the fetus. Imaging tests should be offered to the patient, but they should not replace the evaluation with physical and colposcopic examination. The assessment of fetal well being should be routine and preferably practiced at a high-risk prenatal service. The concern on type of delivery, vaginal delivery should be avoided, and caesarean section should be preferred because vaginal delivery in cases of advanced cervical cancer might increase the risk of lymphatic spread, infections, cervical lacerations and episiotomy-related metastasis ${ }^{8}$. The surgical and postoperative care of the patient should be the same as the oncology patient. According to literature, breastfeeding should be discouraged in patients undergoing treatment with cisplatin ${ }^{9}$. 